BRAZILIAN ARCHIVES OF BIOLOGY AND TECHNOLOGY

AN INTERNATIONAL JOURNAL

\title{
Antidiabetic Potential of Potentilla fulgens Roots in Validated Animal Models of Diabetes
}

\author{
Savita Pal ${ }^{1}$, Arvind Mishra ${ }^{1}$, Arun Kumar Rawat ${ }^{1}$, Sudeep Gautam ${ }^{1}$, Rakesh Maurya ${ }^{2}$ and \\ Arvind K. Srivastava ${ }^{*}$ \\ ${ }^{I}$ Division of Biochemistry, CSIR-Central Drug Research Institute, Jankipuram extension Lucknow. Uttar Pradesh, \\ India. ${ }^{2}$ Division of Medicinal and Process Chemistry, CSIR-Central Drug Research Institute, Jankipuram extension \\ Lucknow. Uttar Pradesh, India.
}

\begin{abstract}
The present study was undertaken to investigate the antidiabetic potential of tap roots of Potentilla fulgens in streptozotocin induced diabetic rat models. The crude powder, ethanolic, ethanolic: aqueous and aqueous extracts of tap roots were administered to normoglycemic-and streptozotocin (STZ)-induced diabetic rats in a single dose study. The ethanolic extract showed significant improvement in oral glucose tolerance and antihyperglycemic effect on sucrose loaded normal rats and STZ-induced diabetic rats. Of the isolated aqueous, $n$-butanol, chloroform and $\mathrm{n}$-hexane soluble fractions of the active ethanolic extract of the roots, the aqueous fraction (100 $\mathrm{mg} / \mathrm{kg}$ body weight) showed significant blood glucose lowering effect on STZ-induced diabetic rats. In a multiple dose study, aqueous fraction of ethanolic extract of $\mathrm{P}$. fulgens roots significantly improved the body weight, percent glycated hemoglobin (\%HbAlc), fasting blood glucose, oral glucose tolerance (OGTT), serum insulin, lipid profile, liver and kidney parameters in STZ-induced diabetic rats. The aqueous fraction also showed marked improvement in OGTT and serum insulin level in neonatal STZ-induced diabetic rats for 30 consecutive days. The aqueous fraction of the roots also inhibited the activity of alpha $(\alpha)$-glucosidase enzyme in a dose dependent manner. In conclusion, the finding suggested that an aqueous fraction of tap roots of $\mathrm{P}$. fulgens possessed potential antidiabetic activity.
\end{abstract}

Keywords: Antidiabetic activity, Antihyperglycemic Activity, Antidyslipidemic activity, Potentilla fulgens roots, alpha $(\alpha)$-glucosidase enzyme

*Authors for correspondence: drarv1955cdri@gmail.com 


\section{INTRODUCTION}

Diabetes mellitus (DM) is globally a major public health concern and represents a main epidemic of the 21th century. The currently available treatment of diabetes includes insulin therapeutics and oral antidiabetic agents, which, however, are effective in mode of action but produce a number of serious adverse effects. Therefore, in this direction, plantbased medicine seems a promising and potential source with safer and lesser, or no side effect over the synthetic drugs in the treatment of diabetes (Sharma et al. 2009; Rai et al. 2011; Singh and Sharma 2013; Watal et al. 2014). Potentilla fulgens Wall. ex Hook. (Family: Rosaceae) is a local medicinal plant, commonly known as bajrandanti, found in temperate, arctic, and alpine zones of the Northen hemisphere, and at higher altitudes of the Khasi Hills, Meghalaya in India. Pharmacological studies have reported that $P$. fulgens possesses anti-hyperglycemic, hypoglycemic, antihyperlipidemic, and antioxidant properties, which supports its ethnotherapeutic uses (Syiem et al. 2009; Jaitak et al. 2010 a). The multiple dose effect of methanol extract of $P$. fulgens roots has hypoglycemic, antihyperglycemic and anti-hyperlipidemic activities in normal mice and alloxan-induced diabetic mice (Syiem et al. 2002; Syiem et al. 2009). Methanol extract selectively enhanced hepatic hexokinase activity (Syiem et al. 2009). The fractions and the isolated compounds of methanol extract of $P$. fulgens roots also have antioxidant activities due to its bioflavonoid, potifulgene and epicatechin compounds (Jaitak et al. $2010 \mathrm{~b}$ ). The information for the antidiabetic effect of isolated fractions of $P$. fulgens roots against the validated animal models of diabetes are few to experimentally validate the possible active fraction with antidiabetic property. Therefore, the present study focused to investigate the antidiabetic effect of the aqueous fraction of tap roots of $P$. fulgens in validated diabetic animal models.

\section{MATERIAL AND METHODS}

\section{Chemicals}

Streptozotocin, metformin, and glybenclamide were purchased from Sigma Chemical Company, St. Louis, USA. The gum acacia, sucrose and glucose were purchased from Sisco Research Laboratory (India).

\section{Animals}

Male rats (12-16 weeks age) of Sprague Dawley (SD) strains weighing between $140 \pm 20 \mathrm{~g}$ of were procured from the animal colony of the National Animal Laboratory Centre (NALC) of Central Drug Research Institute (CDRI), Lucknow, India. The animal experiments were conducted after obtaining institutional ethics committee clearance, in accordance with the guidelines of the Committee for the Purpose of Control and Supervision of Experiments on Animals (CPCSEA) formed by the Government of India in 1964 (Ethics Committee Approval Reference No. 67/13/Biochem/IAEC dated. 12/06/2013). Animals were housed in polypropylene cages in groups of 3 to 5 in the animal room at $23 \pm 2{ }^{\circ} \mathrm{C}$ and relative humidity of $50-60 \%$ under $12: 12 \mathrm{~h}$ light:dark cycle. The animals were provided pellet diet and water ad libitum.

Plant material, Extraction and Fractionation from crude powder of roots of Potentilla fulgens Tap roots of $P$. fulgens were purchased from the local market and identified by the botanist of CDRI, Lucknow. The shade dried material was cut into fine pieces and powdered by a mechanical grinder, passed through 100 mesh sieve and the powder was stored in airtight containers until used. The powder of roots of $P$. fulgens was percolated five times in ethanol at room temperature for $48 \mathrm{~h}$. The filtration and concentration of the combined ethanolic extract was carried out under reduced pressure at $55^{\circ} \mathrm{C}$ in a rota-vapor and the dried substance was termed as ethanolic extract. The dried ethanolic extract was suspended with hexane and hexane insoluble fraction was further suspended with chloroform, butanol and water. The solvents were removed under reduced pressure to furnish corresponding fractions of hexane, chloroform, n-butanol and aqueous. The ethanol extract and all the fractions were stored at $-20^{\circ} \mathrm{C}$ until used.

Assessment of improvement on oral glucose tolerance and blood glucose lowering activity (i) Sucrose loaded normal rats 
Male albino SD rats $(140 \pm 20 \mathrm{~g})$ were selected and kept on an overnight starvation. Next morning, the blood glucose level ( $0 \mathrm{~min}$ ) of each animal was measured by glucometer using glucostrips. The animals showing their fasting blood glucose levels in the range of $60-80 \mathrm{mg} / \mathrm{dL}$ were selected and divided into one control group and five experimental groups with six animals in each $(\mathrm{Pal}$ et al. 2015). Each rat of experimental groups was given suspension of the test substances made in $1.0 \%$ gum acacia at a dosage of $250 \mathrm{mg} / \mathrm{kg}$ bw in the case of crude powder and extracts of $P$. fulgens roots and $25 \mathrm{mg} / \mathrm{kg}$ in the case of standard antidiabetic drug glybenclamide. The animals of the control group received vehicle $(1.0 \%$ gum acacia) only. Exactly 30 min post-administration of the test samples/vehicle, an oral sucrose load of $10 \mathrm{~g} / \mathrm{kg}$ bw was given to each animal and the blood glucose level of each animal were measured at 30, 60, 90 and $120 \mathrm{~min}$.

\section{(ii) Streptozotocin-induced diabetic rats}

Male albino rats of SD strain $(140 \pm 20 \mathrm{~g})$ were selected for this study. Diabetes was induced in the rats by intraperitoneally (i.p.) injecting freshly prepared solution of STZ in $0.1 \mathrm{M}$ citrate buffer $(\mathrm{pH} 4.5)$ at a dosage of $60 \mathrm{mg} / \mathrm{kg}$ body weight (bw). The blood glucose of each animal was checked after $72 \mathrm{~h}$ and animals showing fasting blood glucose values between $280-450 \mathrm{mg} / \mathrm{dL}$ were selected. These diabetic rats were randomly divided into groups consisting of six animals in each. The animals of the experimental groups were orally given the fine suspension of crude powder or extracts $(250 \mathrm{mg} / \mathrm{kg})$ and fractions of roots of $P$. fulgens $(100 \mathrm{mg} / \mathrm{kg} \mathrm{bw})$. The standard antidiabetic drug metformin was given at $100 \mathrm{mg} / \mathrm{kg}$ bw. The diabetic control group received vehicle $(1 \%$ gum acacia) only. Blood samples were collected by tail nipping and blood glucose level was checked by glucostrips at 30,60,90,120,180, 240, 300, and 1440 min, respectively (Pal et al. 2015).

Multiple dose effect of aqueous fraction of roots of $P$. fulgens on streptozotocin-induced diabetic rats

Streptozotocin induced diabetic rats with marked elevation in fasting blood glucose levels above 280-450 mg/dl were used for this study. In order to develop secondary complications associated with chronic diabetes mellitus, STZ-induced diabetic animals were left untreated for a 10 -week period. The diabetic animals, which showed percent glycated hemoglobin level (\%HbA1c) level above 8 on 0 day were recruited in the study. A grouping of six rats in each was done. Group 1 served as a normal control (non-diabetic), Group 2 as a diabetic control and received $1 \%$ gum acacia as a vehicle, whereas, Group 3 and Group 4 received the aqueous fraction of ethanolic extract of roots of $P$. fulgens and metformin $(100 \mathrm{mg} / \mathrm{kg} \mathrm{bw})$ for a period of 30 days. On day $0,7,14,21$, and 28 , the fasting blood glucose level of each animal was determined, followed by performing of an oral glucose tolerance test of each rat at a dosage of 3.0 $\mathrm{gm} / \mathrm{kg}$ post glucose load. The blood was withdrawn from the retro-orbital plexus of each rat on day 10, 20 and 30 for the estimation of serum triglycerides (TG), total cholesterol (TC), low density lipoprotein cholesterol (LDL-C), high density lipoprotein cholesterol (HDL-C), alanine amino transferase (ALT), aspartate amino transferase (AST), urea, uric acid, creatinine and HbA1c levels using assay kits from Dialab diagnostic on Dialab, DTN-410-K Clinical chemistry analyzer. The serum insulin content was determined using the Elisa kit (Mercodia, Uppsala, Sweden).

Effect of aqueous fraction of roots of $P$. fulgens on oral glucose tolerance (OGTT) and serum insulin levels in Neonatally-streptozotocin treated diabetic rats

Two-day-old pups of SD strain (7-10 g) were selected for this experiment. Diabetes was induced in the pups by injecting STZ i.p. (90 mg/kg), prepared in $0.1 \mathrm{M}$ citrate buffer. Non-diabetic control group received only buffer i.p. After four weeks of age, rats were separated from their mothers and acclimatized with free access to food and water. After 12 weeks, diabetes was identified by polydipsia, polyuria, and by performing OGTT. Rats showing glucose intolerance were included in this study and diabetic rats were divided into four groups with six animals in each. The group I and II (non diabetic and diabetic control groups) received vehicle (1\% gum acacia), Group III as an experimental group received a test sample (100 $\mathrm{mg} / \mathrm{kg}$ bw) and Group IV received standard drug metformin $(100 \mathrm{mg} / \mathrm{kg} \mathrm{bw})$. The OGTT of each animal in each group was determined on day 0,7 , 14, 21 and 28 post-treatment. On day 0, 10, 20 and 30 , the blood was withdrawn from animals and the serum was separated and analyzed for the estimation of insulin level. 


\section{Assessment of $\alpha$-glucosidase enzyme inhibition activity}

This was done according to the procedure reported by Pistia-Brueggeman et al. (2001) with a slight modification. A reaction mixture containing 500 $\mu \mathrm{L}$ of phosphate buffer $(0.05 \mathrm{M} ; \mathrm{pH} 6.8), 100 \mu \mathrm{L}$ of purified intestinal $\alpha$-glucosidase $(1.0 \mathrm{U} / \mathrm{mL})$ was pre-incubated at $37^{\circ} \mathrm{C}$ for 1 min with $100 \mu \mathrm{L}$ of glutathione $(1.0 \mathrm{mg} / \mathrm{mL})$ and the desired concentrations of the test sample or standard inhibitor acarbose and the final volume was made to $1000 \mu \mathrm{L}$. Then reaction was started by the addition of $100 \mu \mathrm{L}$ of $p$-nitrophenyl- $\alpha$-Dglucopyranoside (pNPG) $\left(1.0 \times 10^{-3} \mathrm{M}\right)$, substrate of $\alpha$-glucosidase enzyme. The mixture was further incubated at $37^{\circ} \mathrm{C}$ for $20 \mathrm{~min}$ and then the reaction was terminated by the addition of $500 \mu \mathrm{L}$ of $0.1 \mathrm{M}$ sodium carbonate. $\alpha$-glucosidase activity was determined spectrophotometrically at $405 \mathrm{~nm}$ by measuring the quantity of para-nitrophenol released from pNPG. The concentration of the aqueous fraction of $P$. fulgens required to inhibit $50 \% \alpha$-glucosidase activity under the assay conditions was defined as the $\mathrm{IC}_{50}$ value. Experiments were done in triplicates. The inhibition percentage $(\%)$ was calculated by the equation: Inhibition $(\%)=\left(\mathrm{A}_{\text {sample }} / \mathrm{A}_{\text {control }}\right)^{*} 100$ 100.

\section{Statistical analysis}

The results were expressed as mean $\pm \mathrm{SE}$ of three independent experiments. Statistical analysis of each parameter between the groups was done by Dunnett's multiple tests. If the $p$ values were less than $0.05,0.01$ and 0.001 , the results were considered statistically significant, more significant and most significant, respectively.

\section{RESULTS}

Effect of ethanolic extract of $P$. fulgens roots on oral glucose tolerance of sucrose loaded normal rats and blood glucose levels of STZ-induced diabetic rats

The ethanolic extract of $P$. fulgens roots showed significant improvement $(16.4 \%, p<0.01)$ on oral glucose tolerance post-sucrose loaded normoglycemic rats at $250 \mathrm{mg} / \mathrm{kg}$ during $0-120$ min, which was lower than of glybenclamide, which showed around $30.3 \%$ improvement at 25 $\mathrm{mg} / \mathrm{kg}(p<0.01$, Fig. 1 A). A single dose treatment of ethanolic extract of $P$. fulgens at $250 \mathrm{mg} / \mathrm{kg}$ dose to STZ-induced caused lowering on the blood glucose profile to the average of $29.9 \%$ during 0 $300 \mathrm{~min}$ (Fig. 1B) and $22.6 \%$ during 0-1440 min (Fig. 1C). The effect of ethanolic extract of $P$. fulgens roots was comparable to the blood glucose lowering effect of the standard antidiabetic drug, metformin, which showed around 28.6 and $32.6 \%$ lowering in blood glucose level during 0-300 min (Fig. 1B) and 0-1440 min (Fig. 1C), respectively on STZ-induced diabetic rats.

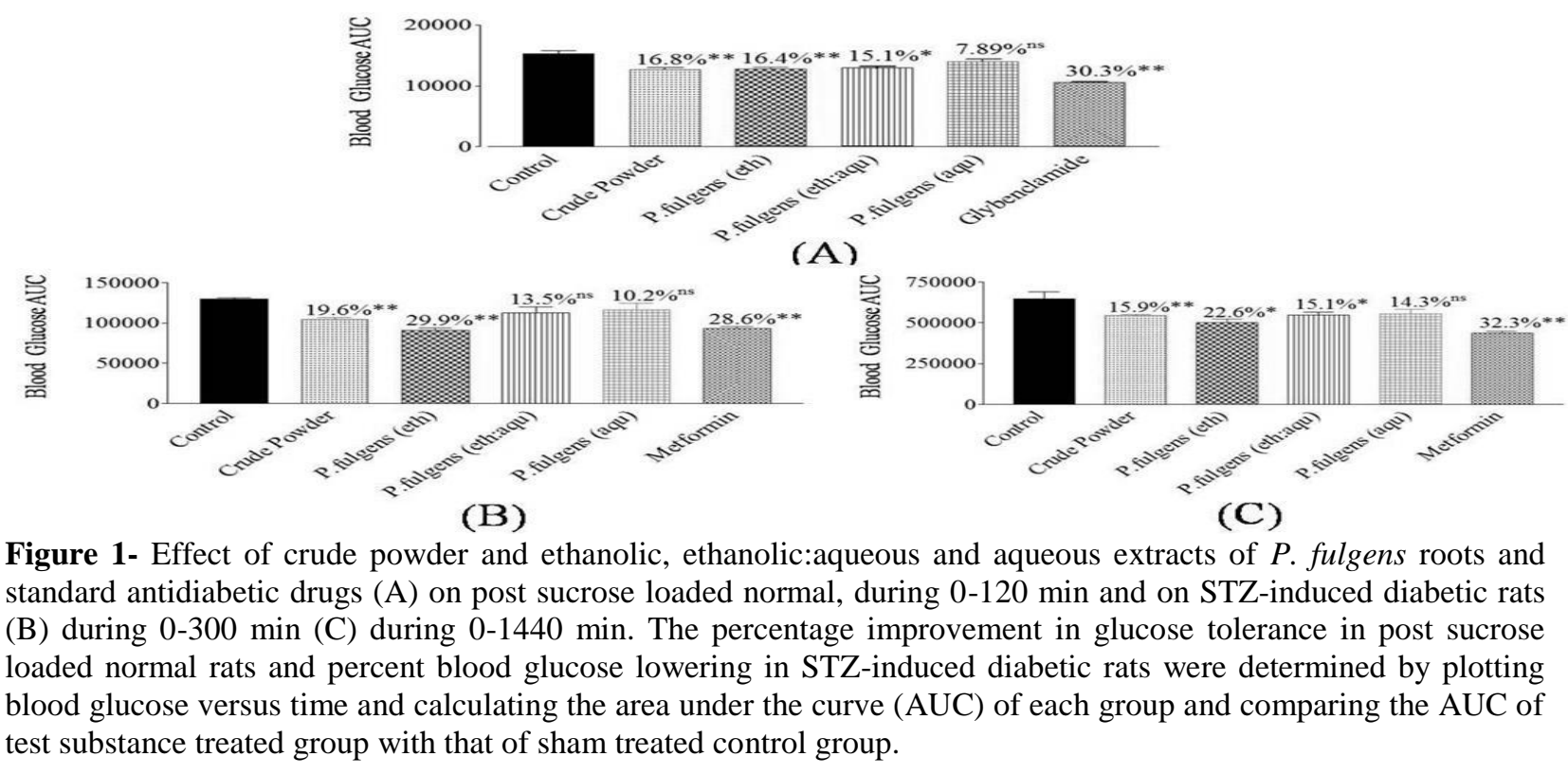


Effect of fractions of ethanolic extract of $P$. fulgens roots on blood glucose levels of STZ induced diabetic rats

Of the hexane, chloroform, butanol and aqueous fractions, aqueous fraction of $P$. fulgens roots showed maximum blood glucose lowering effect on STZ-induced diabetic rats (Figs. 2A-B). The aqueous fraction showed around 33.8 and $28.2 \%$

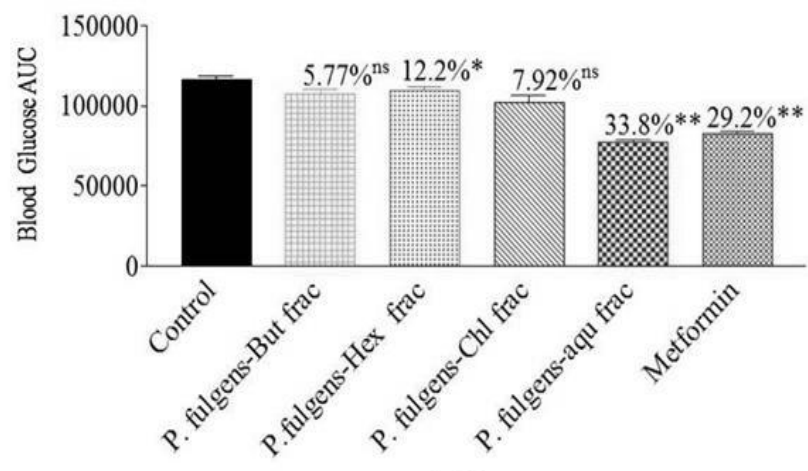

(A) decline in blood glucose levels during 0-300 and 0-1440 min post-treatment, respectively. The almost similar effect was produced by Metformin at dosage $100 \mathrm{mg} / \mathrm{kg}$ body weight, which caused around 29.2 and $32.0 \%$ decline in blood glucose levels during 0-300 and 0-1440 min, respectively (Figs. 2A-B).

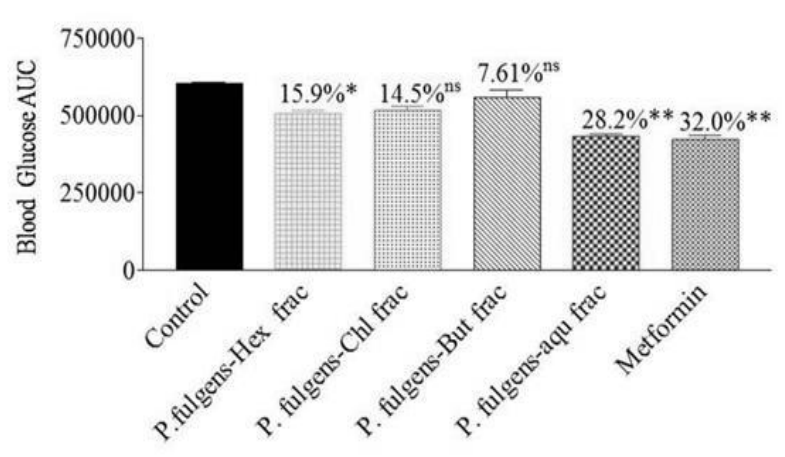

(B)

Figure 2- Effect of Butanol (But), Hexane (Hex), Chloroform (Chl) and aqueous (aqu) fractions (frac) of ethanolic extract of $P$. fulgens roots and standard antidiabetic drug, metformin on blood glucose levels of STZ induced diabetic rats (A) during 0-300 min (B) during 0-1440 min.

Multiple dose effect of aqueous fraction of $P$. fulgens roots on STZ-induced diabetic rats Effect on body weight and \% HbA1c level The administration of the aqueous fraction of ethanolic extract of $P$. fulgens roots to STZ- induced diabetic rats for 30 consecutive days caused a significant improvement in body weight and reduction in elevated level of \% HbAlc level of STZ-induced diabetic rats (Table 1).

Table 1 - Effect of aqueous fraction ethanolic extract of $P$. fulgens roots and standard drug metformin on body weight and \%HbA1c of STZ induced diabetic rats.

\begin{tabular}{|c|c|c|c|c|}
\hline \multirow[t]{2}{*}{ Groups } & \multicolumn{2}{|c|}{ Body Weight (g) } & \multicolumn{2}{|l|}{ HbA1c $(\%)$} \\
\hline & $\begin{array}{l}\text { Initial } \\
\text { (Day 0) }\end{array}$ & $\begin{array}{l}\text { Final } \\
\text { (Day 30) }\end{array}$ & $\begin{array}{l}\text { Initial } \\
\text { (Day 0) }\end{array}$ & $\begin{array}{l}\text { Final } \\
\text { (Day 30) }\end{array}$ \\
\hline $\begin{array}{l}\text { Normal Control } \\
(1.0 \% \text { Gum acacia) }\end{array}$ & $182.7 \pm 2.67$ & $196.0 \pm 3.99$ & $4.87 \pm 0.599$ & $4.90 \pm 0.33$ \\
\hline 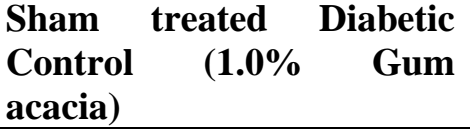 & $152.6 \pm 3.46$ & $139.8 \pm 5.03$ & $11.9 \pm 0.84$ & $12.4 \pm 1.14$ \\
\hline $\begin{array}{l}\text { Aqueous fraction of } 95 \% \\
\text { ethanolic extract of } P \text {. } \\
\text { fulgens roots treated }(100 \\
\text { mg/kg p.o) }\end{array}$ & $149.6 \pm 4.05$ & $\begin{array}{l}164.0 \pm 4.73 \\
(+17.6) * *\end{array}$ & $11.7 \pm 1.90$ & $\begin{array}{l}6.28 \pm 0.282 \\
(49.3)^{* *}\end{array}$ \\
\hline $\begin{array}{l}\text { Metformin treated } \\
(100 \mathrm{mg} / \mathrm{kg} \mathrm{p.o})\end{array}$ & $150.3 \pm 3.90$ & $\begin{array}{l}166.7 \pm 3.32 \\
(+19.2) * *\end{array}$ & $11.9 \pm 0.98$ & $\begin{array}{l}5.94 \pm 0.293 \\
(52.1) * * *\end{array}$ \\
\hline
\end{tabular}


Effect on fasting blood glucose levels and oral glucose tolerance (OGTT)

Evidently the aqueous fraction of $P$. fulgens on fasting blood glucose lowering and improvement of OGTT of STZ induced diabetic was dose dependent, which increased with the days of treatment (Table 2).

Table 2 - Effect of aqueous fraction of P. fulgens roots and standard drug, metformin on fasting blood glucose, and OGTT of STZ induced diabetic rats

\begin{tabular}{|c|c|c|c|c|c|c|c|c|c|c|}
\hline \multirow[t]{2}{*}{ Groups } & \multicolumn{5}{|c|}{$\begin{array}{l}\text { Fasting blood glucose } \\
(\mathbf{m g} / \mathbf{d L})\end{array}$} & \multicolumn{2}{|c|}{$\begin{array}{l}\text { OGTT } \\
0-120 \text { min } \\
(\mathrm{AUC} \pm \mathrm{SEM})\end{array}$} & \multirow[b]{2}{*}{$\begin{array}{l}\text { Day } \\
14\end{array}$} & \multirow[b]{2}{*}{$\begin{array}{l}\text { Day } \\
21\end{array}$} & \multirow[b]{2}{*}{$\begin{array}{l}\text { Day } \\
28\end{array}$} \\
\hline & Day 0 & Day 7 & Day14 & $\begin{array}{l}\text { Day } \\
21\end{array}$ & $\begin{array}{l}\text { Day } \\
28\end{array}$ & Day 0 & Day 7 & & & \\
\hline $\begin{array}{l}\text { Normal Control } \\
(\mathbf{1 . 0 \%} \text { Gum acacia) }\end{array}$ & $\begin{array}{l}74.0 \pm \\
2.73\end{array}$ & $\begin{array}{l}88.6 \pm \\
2.62\end{array}$ & $\begin{array}{l}89.3 \pm 2 \\
.66\end{array}$ & $\begin{array}{l}89.8 \pm 2 \\
.55\end{array}$ & $\begin{array}{l}90.7 \pm \\
2.92\end{array}$ & $\begin{array}{l}12580 \\
\pm 207 \\
8\end{array}$ & $\begin{array}{l}12710 \\
\pm 144.7\end{array}$ & $\begin{array}{l}12950 \\
\pm 126.7\end{array}$ & $\begin{array}{l}12860 \\
\pm 126.6\end{array}$ & $\begin{array}{l}13080 \\
\pm 92.1 \\
0\end{array}$ \\
\hline $\begin{array}{lr}\text { Sham } & \text { treated } \\
\text { Diabetic } & \text { Control } \\
(1.0 \% \text { Gum acacia })\end{array}$ & $\begin{array}{l}384.2 \\
\pm 7.09\end{array}$ & $\begin{array}{l}389.2 \\
\pm 7.13\end{array}$ & $\begin{array}{l}394.0 \pm \\
7.57\end{array}$ & $\begin{array}{l}399.7 \pm \\
6.73\end{array}$ & $\begin{array}{l}401.8 \\
\pm 4.92\end{array}$ & $\begin{array}{l}64710 \\
\pm 446 \\
8\end{array}$ & $\begin{array}{l}64940 \\
\pm 138.3\end{array}$ & $\begin{array}{l}64740 \\
\pm 204.7\end{array}$ & $\begin{array}{l}63880 \\
\pm 951.9\end{array}$ & $\begin{array}{l}63460 \\
\pm 518 \\
4\end{array}$ \\
\hline $\begin{array}{l}\text { Aqueous fraction of } \\
\text { ethanolic ext. of } P \text {. } \\
\text { fulgens roots treated } \\
(100 \mathrm{mg} / \mathrm{kg} \mathrm{p.o})\end{array}$ & $\begin{array}{l}380.3 \\
\pm 18.9\end{array}$ & $\begin{array}{l}340.8 \\
\pm 12.9 \\
(12.4) \\
*\end{array}$ & $\begin{array}{l}329.7 \pm \\
4.01 \\
(16.4)^{*} \\
*\end{array}$ & $\begin{array}{l}304.5 \pm \\
3.69 \\
(23.8)^{*} \\
*\end{array}$ & $\begin{array}{l}287.8 \\
\pm 18.9 \\
(28.4) \\
* *\end{array}$ & $\begin{array}{l}66520 \\
\pm 678 \\
5\end{array}$ & $\begin{array}{l}57550 \\
\pm 272.4 \\
(11.3)^{*}\end{array}$ & $\begin{array}{l}54720 \\
\pm 1136 \\
(15.4)^{*}\end{array}$ & $\begin{array}{l}50780 \\
\pm 1668 \\
(20.5)^{*} \\
*\end{array}$ & $\begin{array}{l}43740 \\
\pm 564 . \\
6 \\
(31.0) \\
* *\end{array}$ \\
\hline $\begin{array}{c}\text { Metformin treated } \\
(100 \mathrm{mg} / \mathrm{kg} \mathrm{p.o})\end{array}$ & $\begin{array}{l}398.0 \\
\pm 13.3\end{array}$ & $\begin{array}{l}335.0 \\
\pm 10.3 \\
(13.9) \\
*\end{array}$ & $\begin{array}{l}313.2 \pm \\
3.22 \\
(20.6)^{*} \\
*\end{array}$ & $\begin{array}{l}301.2 \pm \\
6.35 \\
(24.6)^{*} \\
*\end{array}$ & $\begin{array}{l}282.7 \\
\pm 14.9 \\
(29.7) \\
* *\end{array}$ & $\begin{array}{l}66370 \\
\pm 459 . \\
7\end{array}$ & $\begin{array}{l}57040 \\
\pm 457.2 \\
(12.2)^{*}\end{array}$ & $\begin{array}{l}53600 \\
\pm 1263 \\
(17.2)^{*} \\
*\end{array}$ & $\begin{array}{l}48140 \\
\pm 1381 \\
(24.7)^{*} \\
*\end{array}$ & $\begin{array}{l}42770 \\
\pm 4576 \\
(30.6) \\
* *\end{array}$ \\
\hline
\end{tabular}

Values are mean \pm SE of six rats in each group. Significance at $p^{*}<0.05, p^{* *}<0.01$ and ns: not significant

Effect on serum insulin, lipid profile, liver and kidney function markers

The results demonstrated that both aqueous fraction of $P$. fulgens roots and metformin treatment for 30 days caused an increase in serum insulin, serum HDL-C levels and decrease in serum TG, TC and LDL-C level of streptozotocininduced diabetic rats (Table 3). However, these rats with metformin treatment did not show any significant effect on their serum triglycerides, cholesterol, LDL-C and HDL-C level (Table 3).

Table 3- Effect of aqueous fraction of $P$. fulgens roots and standard drug metformin on serum insulin levels and lipid profile of STZ-induced diabetic rats

\begin{tabular}{|c|c|c|c|c|c|c|}
\hline \multirow[t]{2}{*}{ Group/Treatment } & \multirow[t]{2}{*}{ Day } & \multicolumn{4}{|c|}{ Biochemical profiles (Serum) } & \multirow[b]{2}{*}{$\begin{array}{l}\text { HDL-C } \\
\text { (mg/dL) }\end{array}$} \\
\hline & & $\begin{array}{l}\text { Insulin } \\
\text { (ng/mL) }\end{array}$ & $\begin{array}{l}\text { TG } \\
(\mathrm{mg} / \mathrm{dL})\end{array}$ & $\begin{array}{l}\text { TC } \\
(\mathrm{mg} / \mathrm{dL})\end{array}$ & $\begin{array}{l}\text { LDL-C } \\
\text { (mg/dL) }\end{array}$ & \\
\hline \multirow{3}{*}{$\begin{array}{l}\text { Normal Control } \\
\text { (1.0\% Gum acacia) }\end{array}$} & $\mathbf{1 0}$ & $0.172 \pm 0.007$ & $64.0 \pm 3.06$ & $56.0 \pm 2.44$ & $31.5 \pm 0.76$ & $39.7 \pm 39.3$ \\
\hline & 20 & $0.169 \pm 0.009$ & $64.5 \pm 5.24$ & $59.0 \pm 1.92$ & $32.2 \pm 0.60$ & $39.3 \pm 0.76$ \\
\hline & 30 & $0.167 \pm 0.018$ & $65.8 \pm 2.41$ & $59.3 \pm 1.87$ & $33.3 \pm 0.49$ & $40.3 \pm 2.90$ \\
\hline treated & 10 & $0.084 \pm 0.001$ & $136.0 \pm 2.58$ & $117.8 \pm 2.00$ & $65.6 \pm 0.80$ & $23.0 \pm 0.80$ \\
\hline \multirow{2}{*}{$\begin{array}{l}\text { Diabetic Control } \\
\text { (1.0\% Gum acacia) }\end{array}$} & 20 & $0.082 \pm 0.003$ & $137.5 \pm 1.61$ & $120.8 \pm 0.99$ & $67.2 \pm 1.01$ & $22.8 \pm 1.60$ \\
\hline & 30 & $0.081 \pm 0.003$ & $139.0 \pm 1.44$ & $122.3 \pm 1.33$ & $68.0 \pm 2.17$ & $22.5 \pm 1.86$ \\
\hline \multirow{2}{*}{$\begin{array}{l}\text { Aqueous fraction of } \\
\text { ethanolic ext. of } P \text {. } \\
\text { fulgens roots treated } \\
(100 \mathrm{mg} / \mathrm{kg} \mathrm{p.o})\end{array}$} & 10 & $\begin{array}{l}0.094 \pm 0.006 \\
(11.9)^{\mathrm{ns}}\end{array}$ & $\begin{array}{l}122.8 \pm 1.7 \\
0(9.71)^{\mathrm{ns}}\end{array}$ & $\begin{array}{l}103.5 \pm 3.85 \\
(12.7)^{\mathrm{ns}}\end{array}$ & $\begin{array}{l}56.8 \pm 1.75 \\
(13.4)^{*}\end{array}$ & $\begin{array}{l}24.5 \pm 1.03 \\
(+4.35)^{\mathrm{ns}}\end{array}$ \\
\hline & 20 & $\begin{array}{l}0.104 \pm 0.006 \\
(26.8)^{* *}\end{array}$ & $\begin{array}{l}110.8 \pm 2.0 \\
4(19.4)^{* * *}\end{array}$ & $\begin{array}{l}94.3 \pm 4.41 \\
(21.6) * *\end{array}$ & $\begin{array}{l}48.2 \pm 2.78 \\
(28.2)^{* *}\end{array}$ & $\begin{array}{l}25.5 \pm 1.77 \\
(+11.7)^{\mathrm{ns}}\end{array}$ \\
\hline
\end{tabular}




\begin{tabular}{|c|c|c|c|c|c|c|}
\hline & 30 & $\begin{array}{l}0.112 \pm 0.002 \\
(38.3)^{* *}\end{array}$ & $\begin{array}{l}95.5 \pm 2.14 \\
(31.3)^{* *}\end{array}$ & $\begin{array}{l}74.3 \pm 5.18 \\
(39.2)^{* *} \\
\end{array}$ & $\begin{array}{l}44.0 \pm 2.28 \\
(35.2)^{* *}\end{array}$ & $\begin{array}{l}25.7 \pm 1.67 \\
(+14.0)^{*}\end{array}$ \\
\hline \multirow[t]{3}{*}{$\begin{array}{l}\text { Metformin treated } \\
(100 \mathrm{mg} / \mathrm{kg} \mathrm{p.o})\end{array}$} & 10 & $\begin{array}{l}0.092 \pm 0.003 \\
(9.52)^{\mathrm{ns}}\end{array}$ & $\begin{array}{l}125.5 \pm 2.4 \\
0(7.72)^{\mathrm{ns}}\end{array}$ & $\begin{array}{l}111.7 \pm 2.06 \\
(5.17)^{\mathrm{ns}}\end{array}$ & $\begin{array}{l}62.2 \pm 1.23 \\
(5.27)^{\mathrm{ns}}\end{array}$ & $\begin{array}{l}23.5 \pm 1.11 \\
(+2.17)^{\mathrm{ns}}\end{array}$ \\
\hline & 20 & $\begin{array}{l}0.095 \pm 0.006 \\
(15.9)^{*}\end{array}$ & $\begin{array}{l}124.3 \pm 1.4 \\
6(9.60)^{\mathrm{ns}} \\
\end{array}$ & $\begin{array}{l}109.8 \pm 3.49 \\
(8.73)^{\mathrm{ns}}\end{array}$ & $\begin{array}{l}61.7 \pm 3.06 \\
(8.19)^{\mathrm{ns}} \\
\end{array}$ & $\begin{array}{l}24.5 \pm 1.52 \\
(+7.31)^{\mathrm{ns}}\end{array}$ \\
\hline & 30 & $\begin{array}{l}0.102 \pm 0.005 \\
(25.9)^{* *}\end{array}$ & $\begin{array}{l}121.2 \pm 2.0 \\
9(12.8)^{\mathrm{ns}}\end{array}$ & $\begin{array}{l}107.0 \pm 3.20 \\
(12.5)^{\mathrm{ns}}\end{array}$ & $\begin{array}{l}60.9 \pm 2.06 \\
(10.4)^{\mathrm{ns}}\end{array}$ & $\begin{array}{l}25.0 \pm 1.51 \\
(+11.1)^{\mathrm{ns}}\end{array}$ \\
\hline
\end{tabular}

Values are mean $\pm \mathrm{SE}$ of six rats in each group. Significance at $p^{*}<0.05, p^{* *}<0.01$ and ns: not significant

It was evident from results that effect of the aqueous fraction of $P$. fulgens roots and metformin caused significant reduction in elevated levels of serum ALT levels, serum AST levels, serum urea, uric acid and creatinine levels of STZ induced diabetic rats with increase in duration of treatment in a dose-dependent manner (Table 4).

Table 4- Effect of aqueous fraction of $P$. fulgens roots and standard drug metformin on liver and kidney functions parameters of STZ-induced diabetic rats.

\begin{tabular}{|c|c|c|c|c|c|c|}
\hline \multirow[t]{3}{*}{ Group/Treatment } & \multirow[t]{3}{*}{ Day } & \multicolumn{5}{|c|}{ Biochemical Parameters (Serum) } \\
\hline & & \multicolumn{2}{|l|}{ Liver } & \multicolumn{3}{|c|}{ Renal Function Markers } \\
\hline & & $\begin{array}{l}\text { ALT } \\
(\mathbf{U} / \mathbf{L})\end{array}$ & $\begin{array}{l}\text { AST } \\
(\mathbf{U} / \mathbf{L})\end{array}$ & $\begin{array}{l}\text { Urea } \\
(\mathbf{m g} / \mathbf{d L})\end{array}$ & $\begin{array}{l}\text { Uric acid } \\
(\mathbf{m g} / \mathbf{d L})\end{array}$ & $\begin{array}{l}\text { Creatinine } \\
(\mathrm{mg} / \mathrm{dL})\end{array}$ \\
\hline \multirow{4}{*}{$\begin{array}{l}\text { Normal Control } \\
(1.0 \% \text { Gum acacia) }\end{array}$} & 10 & $12.8 \pm 0.61$ & $13.9 \pm 1.13$ & $23.2 \pm 1.14$ & $2.05 \pm 0.17$ & $0.39 \pm 0.05$ \\
\hline & 20 & $12.8 \pm 0.60$ & $14.1 \pm 1.08$ & $24.2 \pm 1.01$ & $2.05 \pm 0.28$ & $0.39 \pm 0.02$ \\
\hline & 30 & $13.7 \pm 0.76$ & $14.5 \pm 1.13$ & $24.5 \pm 0.88$ & $2.08 \pm 0.19$ & $0.40 \pm 0.2$ \\
\hline & $\mathbf{0}$ & $82.0 \pm 4.07$ & $88.3 \pm 3.20$ & $85.5 \pm 2.41$ & $8.42 \pm 0.72$ & $0.83 \pm 0.04$ \\
\hline \multirow{3}{*}{ 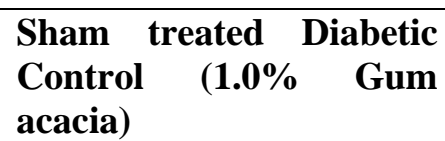 } & 10 & $81.8 \pm 2.54$ & $89.0 \pm 2.82$ & $86.2 \pm 1.96$ & $8.55 \pm 0.73$ & $0.83 \pm 0.04$ \\
\hline & 20 & $84.0 \pm 2.49$ & $89.2 \pm 2.73$ & $88.0 \pm 2.29$ & $8.59 \pm 0.67$ & $0.86 \pm 0.05$ \\
\hline & $\mathbf{3 0}$ & $86.7 \pm 1.86$ & 89.2 & $88.2 \pm 2.12$ & $8.66 \pm 0.68$ & $=0.03$ \\
\hline \multirow{3}{*}{ 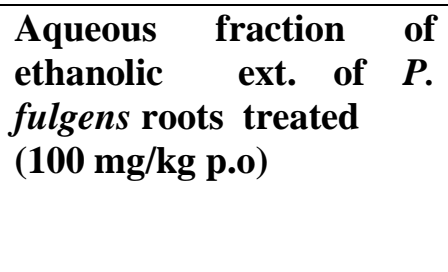 } & 10 & $\begin{array}{l}72.3 \pm 2.06 \\
(11.6)^{\mathrm{ns}}\end{array}$ & $\begin{array}{l}79.0 \pm 4.25 \\
(11.2)^{\mathrm{ns}}\end{array}$ & $\begin{array}{l}78.3 \pm 3.25 \\
(9.09)^{\mathrm{ns}}\end{array}$ & $\begin{array}{l}7.28 \pm 0.74 \\
(14.8)^{\mathrm{ns}}\end{array}$ & $\begin{array}{l}0.77 \pm 0.02 \\
(6.38)^{\mathrm{ns}}\end{array}$ \\
\hline & 20 & $\begin{array}{l}66.2 \pm 3.52 \\
(21.2)^{* *}\end{array}$ & $\begin{array}{l}75.7 \pm 3.16 \\
(15.1)^{*}\end{array}$ & $\begin{array}{l}74.8 \pm 2.22 \\
(14.9)^{*}\end{array}$ & $\begin{array}{l}5.93 \pm 0.20 \\
(31.0)^{* *}\end{array}$ & $\begin{array}{l}0.72 \pm 0.03 \\
(16.3)^{*}\end{array}$ \\
\hline & 30 & $\begin{array}{l}57.2 \pm 4.06 \\
(34.0)^{* *}\end{array}$ & $\begin{array}{l}64.7 \pm 2.52 \\
(24.5)^{* *}\end{array}$ & $\begin{array}{l}69.2 \pm 0.87 \\
(21.5)^{* *}\end{array}$ & $\begin{array}{l}4.87 \pm 0.29 \\
(43.7) * *\end{array}$ & $\begin{array}{l}0.63 \pm 0.02 \\
(26.6) * *\end{array}$ \\
\hline \multirow[t]{3}{*}{$\begin{array}{l}\text { Metformin } \\
(100 \mathrm{mg} / \mathrm{kg} \text { p.o })\end{array}$} & 10 & $\begin{array}{l}72.5 \pm 2.99 \\
(11.4)^{\mathrm{ns}}\end{array}$ & $\begin{array}{l}81.0 \pm 4.48 \\
(8.99)^{\mathrm{ns}}\end{array}$ & $\begin{array}{l}79.8 \pm 2.60 \\
(7.36)^{\mathrm{ns}}\end{array}$ & $\begin{array}{l}7.32 \pm 0.54 \\
(14.4)^{\mathrm{ns}}\end{array}$ & $\begin{array}{l}0.75 \pm 0.05 \\
(9.90)^{\mathrm{ns}}\end{array}$ \\
\hline & 20 & $\begin{array}{l}61.5 \pm 2.62 \\
(26.8)^{* *}\end{array}$ & $\begin{array}{l}77.8 \pm 3.93 \\
(12.7)^{\mathrm{ns}}\end{array}$ & $\begin{array}{l}77.0 \pm 5.02 \\
(12.5)^{\mathrm{ns}}\end{array}$ & $\begin{array}{l}6.29 \pm 0.33 \\
(26.7) * *\end{array}$ & $\begin{array}{l}0.74 \pm 0.03 \\
(13.5)^{\mathrm{ns}}\end{array}$ \\
\hline & 30 & $\begin{array}{l}57.3 \pm 2.39 \\
(33.8)^{* *}\end{array}$ & $\begin{array}{l}68.0 \pm 2.82 \\
(23.7) * *\end{array}$ & $\begin{array}{l}69.3 \pm 1.72 \\
(21.4)^{* *}\end{array}$ & $\begin{array}{l}5.13 \pm 0.60 \\
(40.7)^{* *}\end{array}$ & $\begin{array}{l}0.59 \pm 0.05 \\
(31.1)^{* *}\end{array}$ \\
\hline
\end{tabular}

Values are mean \pm SE of six rats in each group. Significance at $p^{*<0.05}, p^{* *<0.01}$ and ns: not significant

Effect of the aqueous fraction of $P$. fulgens roots on fasting blood glucose, OGTT and serum insulin level of neonatally STZ induced diabetic rats

The results indicated that both aqueous fraction of $P$. fulgens roots and metformin treatment to neonatally-STZ treated rats caused a significant decline in their fasting blood glucose profile and improvement of OGTT from day 7 to day 28
(Table 5). Whereas, the serum insulin levels in both aqueous fraction and metformin treated neonatally STZ-induced diabetic rats increased after 30 days of treatment (Fig. 3). 


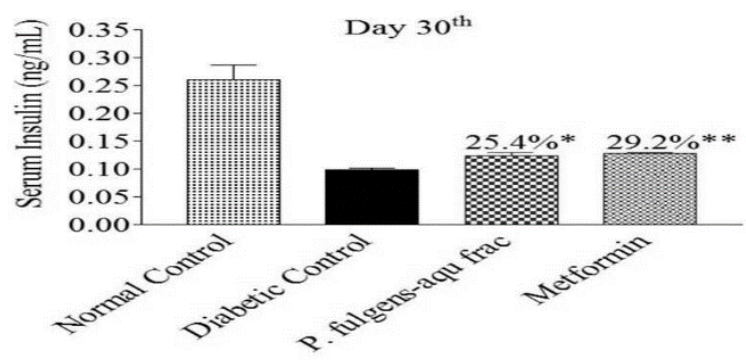

Figure 3 - Effect of aqueous fraction (aqu frac) of $P$. fulgens roots and standard drug metformin on serum insulin profile of neonatally STZ induced diabetic rats. $\mathrm{p}^{* *}<0.01$

Table 5 - Effect of aqueous fraction of $P$. fulgens roots and metformin on fasting blood glucose and OGTT of neonatally STZ-induced diabetic rats

\begin{tabular}{|c|c|c|c|c|c|c|c|c|c|c|}
\hline \multirow[t]{2}{*}{ Group/Treatment } & \multicolumn{5}{|c|}{$\begin{array}{l}\text { Fasting blood glucose } \\
(\mathrm{mg} / \mathrm{dL})\end{array}$} & \multicolumn{5}{|c|}{$\begin{array}{l}\text { Oral Glucose tolerance } \\
(0-120 \text { min }) \\
(\text { AUC } \pm \text { SEM })\end{array}$} \\
\hline & $\begin{array}{l}\text { Day } \\
\text { 0 }\end{array}$ & $\begin{array}{l}\text { Day } \\
7\end{array}$ & $\begin{array}{l}\text { Day } \\
14\end{array}$ & $\begin{array}{l}\text { Day } \\
21\end{array}$ & $\begin{array}{l}\text { Day } \\
28\end{array}$ & $\begin{array}{l}\text { Day } \\
0\end{array}$ & $\begin{array}{l}\text { Day } \\
7\end{array}$ & $\begin{array}{l}\text { Day } \\
14\end{array}$ & $\begin{array}{l}\text { Day } \\
21\end{array}$ & $\begin{array}{l}\text { Day } \\
28\end{array}$ \\
\hline $\begin{array}{l}\text { Normal Control } \\
(1.0 \% \text { Gum acacia) }\end{array}$ & $\begin{array}{l}76.9 \pm \\
3.60\end{array}$ & $\begin{array}{l}80.8 \pm \\
2.66\end{array}$ & $\begin{array}{l}80.7 \pm \\
2.89\end{array}$ & $\begin{array}{l}87.9 \pm \\
2.67\end{array}$ & $\begin{array}{l}88.6 \pm \\
3.55\end{array}$ & $\begin{array}{l}12440 \\
\pm 149 \\
1 \\
\end{array}$ & $\begin{array}{l}12723 \\
\pm 157 \\
9 \\
\end{array}$ & $\begin{array}{l}12750 \\
\pm 139 \\
3 \\
\end{array}$ & $\begin{array}{l}12809 \pm \\
130.1\end{array}$ & $\begin{array}{l}12812 \\
\pm 188.0\end{array}$ \\
\hline $\begin{array}{l}\text { Sham treated } \\
\text { Diabetic Control } \\
\text { (1.0\% gum acacia) }\end{array}$ & $\begin{array}{l}191.7 \\
\pm 3.39\end{array}$ & $\begin{array}{l}190.8 \\
\pm 3.59\end{array}$ & $\begin{array}{l}191.3 \\
\pm 3.34\end{array}$ & $\begin{array}{l}192.7 \\
\pm 2.78\end{array}$ & $\begin{array}{l}193.7 \\
\pm 2.26\end{array}$ & $\begin{array}{l}46070 \\
\pm 642 \text {. } \\
5\end{array}$ & $\begin{array}{l}47090 \\
\pm 563 \text {. } \\
9\end{array}$ & $\begin{array}{l}47580 \\
\pm 959 \text {. } \\
1\end{array}$ & $\begin{array}{l}47340 \pm \\
1519.0\end{array}$ & $\begin{array}{l}51920 \\
\pm 1590 \text {. } \\
0\end{array}$ \\
\hline $\begin{array}{l}\text { Aqueous fraction of } \\
\text { ethanolic ext. of } P \text {. } \\
\text { fulgens roots treated } \\
(100 \mathrm{mg} / \mathrm{kg} \mathrm{p.o})\end{array}$ & $\begin{array}{l}191.8 \\
\pm 5.67\end{array}$ & $\begin{array}{l}160.8 \\
\pm 8.33 \\
(15.7 \\
)^{*}\end{array}$ & $\begin{array}{l}133.0 \\
\pm 3.09 \\
(30.5 \\
)^{* *}\end{array}$ & $\begin{array}{l}111.2 \\
\pm 1.62 \\
(42.3 \\
)^{* *}\end{array}$ & $\begin{array}{l}106.3 \\
\pm 1.82 \\
(45.1 \\
)^{* *}\end{array}$ & $\begin{array}{l}46600 \\
\pm 929 \text {. } \\
0\end{array}$ & $\begin{array}{l}39180 \\
\pm 508 . \\
2 \\
(16.8 \\
)^{* *}\end{array}$ & $\begin{array}{l}35770 \\
\pm 637 . \\
3 \\
(24.8) \\
* *\end{array}$ & $\begin{array}{l}23410 \pm \\
377.5 \\
(50.5)^{*} \\
*\end{array}$ & $\begin{array}{l}21660 \\
\pm 481.0 \\
(58.3) \\
* *\end{array}$ \\
\hline $\begin{array}{l}\text { Metformin } \\
(100 \text { mg/kg p.o) }\end{array}$ & $\begin{array}{l}191.7 \\
\pm 15.6\end{array}$ & $\begin{array}{l}164.0 \\
\pm 2.38 \\
(14.0 \\
)^{* *}\end{array}$ & $\begin{array}{l}129.5 \\
\pm 3.03 \\
(32.3 \\
)^{* *}\end{array}$ & $\begin{array}{l}104.7 \\
\pm 2.24 \\
(45.6 \\
)^{* *}\end{array}$ & $\begin{array}{l}107.2 \\
\pm 1.81 \\
(44.7 \\
)^{* *}\end{array}$ & $\begin{array}{l}46830 \\
\pm 845 \\
1\end{array}$ & $\begin{array}{l}36900 \\
\pm 6027 \\
(21.6) \\
* *\end{array}$ & $\begin{array}{l}33080 \\
\pm 195 . \\
9 \\
(30.5) \\
* *\end{array}$ & $\begin{array}{l}23280 \pm \\
665.6 \\
(50.8)^{*} \\
*\end{array}$ & $\begin{array}{l}21200 \\
\pm 768.7 \\
(59.2) \\
* *\end{array}$ \\
\hline
\end{tabular}

Values are mean \pm S.E. of six rats in each group. $p^{*<0.05}, p^{* *<0.01, n s: n o t ~ s i g n i f i c a n t ~}$

Inhibitory effect of aqueous fraction of $P$. fulgens roots on $\alpha$-glucosidase enzyme

The aqueous fraction of $P$. fulgens roots exerted an inhibitory effect on $\alpha$-glucosidase with $50 \%$ inhibition $\left(\mathrm{IC}_{50}=46.9 \mu \mathrm{g} / \mathrm{mL}\right)$, which was more than the $50 \%$ inhibition exerted by a standard inhibitor, acarbose ( $\left.\mathrm{IC}_{50}=43.3 \mu \mathrm{g} / \mathrm{mL}\right)$ as shown in Figure 4.

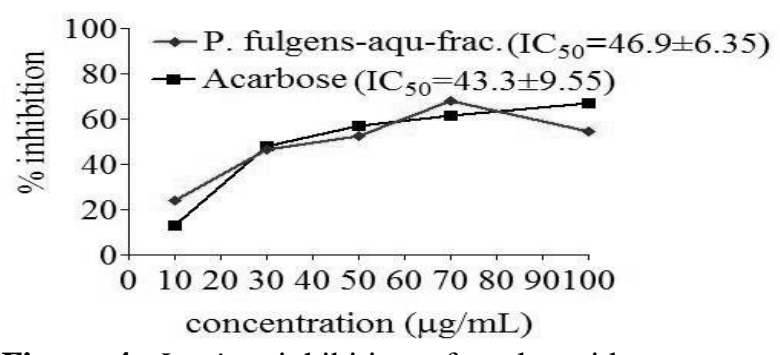

Figure 4- In-vitro inhibition of $\alpha$-glucosidase enzyme by the aqueous fraction of $P$. fulgens roots. $\mathrm{IC}_{50}=$ Values are means $\pm \mathrm{SE}$ of three replicates.

\section{DISCUSSION}

Many new bioactive phytochemicals isolated from the plants having hypoglycemic and antihyperglycemic effects show the same antidiabetic activity and sometimes even more potent than already known oral hypoglycemic agents (Rai et al. 2013; Watal et al. 2014). Keeping this view in consideration, in the present study, the ethanolic, ethanolic: aqueous and aqueous extracts of $P$. fulgens roots were investigated for their oral glucose tolerance as well as the antihyperglycemic effect on sucrose loaded normal rats and STZ-induced diabetic rats. Of all these extracts, the ethanolic extract of $P$. fulgens roots showed most significant improvement in the blood glucose profile of post sucrose loaded 
normal rats and STZ-induced diabetic rats compared to that of standard antidiabetic drugs, glybenclamide and metformin, respectively, indicating augmentation in the peripheral blood glucose utilization, increase in insulin secretion, insulin sensitizer, and insulin mimetic from the pancreatic beta cells, or beta cell regeneration ability, inhibitory potential of the enzyme $\alpha$ glucosidase, which were some of the probable mechanisms of action of ethanolic extract for their antihyperglycemic effect.

The isolated fractions of the ethanolic extract of $P$. fulgens roots were evaluated for their antihyperglycemic effect on STZ-induced diabetic rats. Of these fractions, the aqueous fraction of ethanolic extract of $P$. fulgens roots exhibited significant antihyperglycemic effect on STZ induced diabetic rats. The effect shown by the ethanolic extract/aqueous fraction might be due to their insulin secretogogues, insulin sensitizing, or insulin mimetic activities. These results when compared with metformin, revealed the insulin sensitizing effect of ethanolic extract and its aqueous fraction of $P$. fulgens roots. However, the antidiabetic efficacy and hypoglycemic mechanisms of aqueous fraction of ethanolic extract of $P$. fulgens roots have not been fully defined in different diabetic models. Therefore, in the present study, antihyperglycemic and antidyslipidemic activities of the aqueous fraction of ethanolic extract of $P$. fulgens roots were identified in validated animal models of diabetes mellitus, i.e., STZ-induced diabetic rats, and 2days neonates (n) 2-STZ-induced diabetic rats.

Streptozotocin (STZ, 2-deoxy-2-(3-(meyl-3nitrosoureido)-D-glucopyranose) is obtained from Streptomycetes achromogenes and is used to induce diabetes in animals (Balamurugan et al. 2003). STZ injection is used to develop hyperglycemia and decreases insulin levels in blood (Nabi et al. 2013). STZ-induced diabetic rat model is the most common animal model of human diabetes and have been found suitable to study long-term complications of diabetes. In the present study, STZ-injection caused a significant reduction of body weight, persistent hyperglycemia marked by increase in the level of glycosylated haemoglobin (HbA1c) and decrease in insulin secretion from $\beta$-cells of the pancreas (Rai et al. 2010; Nabi et al. 2013; Chauhan et al. 2015). The oral administration of the aqueous fraction of ethanolic extract of $P$. fulgens roots at (100 mg/kg bw dose) to STZ-induced diabetic rats caused a significant improvement in body weight, reduction in glycated hemoglobin, decline in fasting blood glucose level and improvement in glucose tolerance and also enhanced insulin secretion from $\beta$ cells of the pancreas. In the earlier studies, it has been shown that the oral administration of methanol extract of $P$. fulgens to alloxan induced diabetic mice showed improvement in serum triglyceride, total cholesterol and HDL cholesterol levels (Syiem et al. 2009). In the present study, the aqueous fraction of $P$. fulgens roots showed a significant decrease in TG, TC and LDL-C levels while significant increase in HDL-C levels as compared to sham treated control. There was a significant decrease $(p<0.01)$ in the serum AST and ALT levels in the elevated levels of serum AST and ALT in STZ-induced diabetic rats with the treatment of aqueous fraction of $P$. fulgens roots by consecutively dosing for 30 days. The liver releases ALT and AST, elevation in their plasma concentrations was an indicator of liver damage (Crook 2006; Rai et al. 2008). The treatment of the STZ-induced diabetic rats with the aqueous fraction of $P$. fulgens roots and metformin, both showed improvement in the renal functions as evidenced by the decline in the serum urea, uric acid and creatinine levels of the STZ-induced diabetic rats, thus also improved the kidney functions along with the liver function as described in Table 4. The elevation in the plasma urea, uric acid and creatinine concentrations relates to kidney function impairment specifically the renal filtration mechanism (Pradeepa et al. 2013).

The neonatal STZ induced diabetic rats are considered to be better tools for the elucidation of the mechanisms associated with regeneration of the beta cells, functional exhaustion of the beta cells and emergence of defects in insulin action (Portha et al. 1994; Fernandez-Alvarez et al. 2004;). It has been shown that at 8-10 weeks of age and thereafter, neonatal rats treated with STZ, caused mild basal hyperglycemia, an impaired response to the glucose tolerance test (Islam et al. 2009), and a loss of $\beta$ cell sensitivity to glucose (Giroix et al. 1983). However, in the present study, treatment with an aqueous fraction of $P$. fulgens roots enhanced the serum insulin level in neonatal STZ-induced diabetic adult rats, which might have resulted due to the regeneration of $\beta$-cells of pancreas as compared to sham treated control, and the oral glucose tolerance was also significantly 
improved in the treated neonatal STZ-induced diabetic rats (Fig. 3 and Table 5). These results indicated that the aqueous fraction of ethanolic extract of $P$. fulgens roots was effective in improving glucose intolerance and insulin secretion of the neonatally-STZ-induced diabetic rats. Postprandial hyperglycemia is an early defect of type- 2 diabetes and one of the primary antidiabetic targets. Treatment of postprandial hyperglycemia can be achieved by inhibiting the intestinal $\alpha$-glucosidase, the key enzyme for oligosaccharide digestion and further delayed glucose absorption (Kumar et al. 2011). The phytochemicals such as flavonoids in aqueous preparation of plants have shown to possess antidiabetic and antioxidant activities (Sharma et al. 2009; Jaiswal et al. 2013).

The present study suggested that the aqueous fraction of $P$. fulgens roots might exhibit antidiabetic properties by slowing down the assimilation of carbohydrate, ameliorating postprandial hyperglycemia and increased the insulin sensitivity, mainly due to its ability to inhibit the intestinal $\alpha$-glucosidase activity (Antu et al. 2014). Thus, active compounds could be isolated from an aqueous fraction of $P$. fulgens roots, which might be used as a lead compound for the development of antidiabetic therapeutics.

\section{CONCLUSION}

In conclusion, the aqueous fraction of ethanolic extract of $P$. fulgens roots displayed potential antidiabetic activity by inhibiting $\alpha$-glucosidase enzyme and ameliorating diabetes associated hyperlipidemia increased $\mathrm{HbAlc}$, altered liver, and kidney function markers. The antidiabetic component of the fraction might involve single, or more compounds which ameliorate diabetic features, suggest that the natural constituents could act independently, or synergistically to induce an antihyperglycemic effect. Further pharmacological and biochemical investigations underline the elucidation of the exact mechanism and isolation of its active principle.

\section{ACKNOWLEDGEMENTS}

The authors are thankful to ICMR, New Delhi (India) for providing financial support. The authors also acknowledge Dr. Harjeet Singh Maan, for proofreading the manuscript.

\section{REFERENCES}

Antu KA, Riya MP, Mishra A, Anilkumar KS, Chandrakanth CK, Tamrakar AK, et al. Antidiabetic property of Symplocos cochinchinensis is mediated by inhibition of alpha glucosidase and enhanced insulin sensitivity. PLoS One. 2014 Sep 3; 9(9):e105829.

Balamurugan AN, Miyamoto M, Wang W, Inoue K, Tabata Y. Streptozotocin (STZ) used to induce diabetes in animal models. J Ethnopharm. 2003; 26:102-103.

Chauhan P, Mahajan S, Kulshrestha A, Shrivastava S, Sharma B, Goswamy HM, et al. Bougainvillea spectabilis Exhibits Antihyperglycemic and Antioxidant Activities in Experimental Diabetes. J Evid Based Complementary Altern Med. 2015.

Crook MA. Clinical Chemistry and Metabolic Medicine. Hodder Arnold, London. 2006; 7 Edition. 426.

Farooqui AHA, Sharma S, Khan A, Kumar R and Kumar S. Formulation useful as a natural herbal tooth powder. United States Patent. 2001; 6,264,926, B1, 24:27.

Fernandez-Alvarez J, Barbera A, Nadal B, BarceloBatllori S, Piquer S, Claret $\mathrm{M}$, et al. Stable and functional regeneration of pancreatic beta-cell population in $\mathrm{nSTZ}$ rats treated with tungstate. Diabetologia. 2004; 47: 470-477.

Giroix Mh, Pora B, Kergoat M, Bailbe D, Picon L: Glucose insensitivity and amino-acid hypersensitivity of insulin release in rats with noninsulin-dependent diabetes: a study with the perfused pancreas. Diabetes. 1983; 32: 445-451.

Islam MS, Loots du T. Experimental rodent models of type 2 diabetes: a review. Methods Find Exp Clin Pharmacol. 2009; 31(4):249-261.

Jaiswal D, Rai PK, Mehta S, Chatterji S, Shukla S, Rai DK, et al. Role of Moringa oleifera in regulation of diabetes-induced oxidative stress. Asian Pac J Trop Med. 2013; 6 (6):426-432.

Jaitak V, Kaul VK, Himlata, Kumar N, Singh B, Dhar J et al. New hopane triterpenes and antioxidant constituents from Potentilla fulgens. Nat Prod Commun. 2010b; 5(10):1561-1566.

Jaitak V, Sharma K, Kalia K, Kumar N, Singh HP, Kaul VK et al. Antioxidant activity of Potentilla fulgens : An alpine plant of western Himalaya. J Food Compos Anal. 2010a, 23 (2):142-147.

Kumar S, Narwal S, Kumar V, Prakash O. $\alpha-$ glucosidase inhibitors from plants: A natural approach to treat diabetes. Pharmacogn Rev. 2011; 5(9):19-29.

Nabi SA, Kasetti RB, Sirasanagandla S, Tilak TK, Kumar MV, Rao CA. Antidiabetic and antihyperlipidemic activity of Piper longum root 
aqueous extract in STZ induced diabetic rats. $B M C$ Complement Altern Med. 2013; 13:37.

Pal S, Mishra A, Rawat AK, Maurya R, Srivastava AK. Antidiabetic potential of whole plant of Phyllanthus niruri. World Journal of Pharmaceutical Research. 2015; 4(5): 1010-1042.

Pistia-Brueggeman, G, Hollingswor RI. A preparation and screening strategy for glucosidase inhibitors. Tetrahedron. 2001; 57, 8773-8778.

Portha B, Giroix MH, Serradas P, Morin L, Tormo MA, Bailbe D. Cellular basis for glucose refractoriness of pancreatic $\beta$-cells in non insulin dependent diabetes. In: Flatt PR, Lenzen S, editors. Insulin secretion and pancreatic $\beta$-cell research. UK: Smi-Gordon. 1994; $461-472$

Pradeepa S, Subramanian S, Kaviyarasan V. Biochemical evaluation of antidiabetic properties of Piecellobium dulce fruits studied in streptozotocin induced experimental diabetic rats. Int. J. Herb. Medi. 2013; 1 (4): 21-28.

Rai PK, Jaiswal D, Rai DK, Sharma B, Watal G. Effect of water extract of Trichosanthes dioica fruits in streptozotocin induced diabetic rats. Indian J Clin Biochem. 2008; 23 (4):387-390.

Rai PK, Jaiswal D, Mehta S, Rai DK, Sharma B, Watal G. Effect of Curcuma longa freeze dried rhizome powder with milk in STZ induced diabetic rats. Indian J Clin Biochem. 2010; 25 (2):175-181.

Rai PK, Rai DK, Mehta S, Gupta R, Sharma B, Watal G. Effect of Trichosanthes dioica on oxidative stress and CYP450 gene expression levels in experimentally induced diabetic rats. Cell Mol Biol (Noisy-le-grand). 2011; 57(1):31-9.
Rai PK, Srivastava AK, Sharma B, Dhar P, Mishra AK, Watal G. Use of laser-induced breakdown spectroscopy for the detection of glycemic elements in Indian medicinal plants. Evid Based Complement Alternat Med. 2013; 2013:406365.

Sharma RK, Chatterji S, Rai DK, Mehta S, Rai PK, Singh RK, et al. Antioxidant activities and phenolic contents of the aqueous extracts of some Indian medicinal plants. J Med Plants Res. 2009; 3(11): 944-948.

Singh RK and Sharma B. Certain Traditional Indian Plants and Their Therapeutic Applications: A Review. Vri Phytomedicine. 2013; 1 (1): 1-11.

Syiem D, Khup PZ and Syiem AB. Effects of Potentilla fulgens Linn. on carbohydrate and lipid profiles in diabetic mice. Pharmacologyonline. 2009; 2: 787795.

Syiem D, Syngai G, Khup PZ, Khongwir BS, Kharbuli B, Kayang H. Hypoglycemic effects of Potentilla

fulgens L. in normal and alloxan-induced diabetic mice. J Ethnopharmacol. 2002; 83 (1-2): 55-61.

Watal G, Dhar P, Srivastava SK, Sharma B. Herbal medicine as an alternative medicine for treating diabetes: the global burden. Evid Based Complement Alternat Med. 2014; 2014:596071.

Received: September 27, 2015; Accepted: April 01, 2016. 